\title{
LabVIEW Based Instrument Cluster Tester
}

\author{
Deeksha K ${ }^{1}$, Sandeep Reddy Cherukupalli ${ }^{2}$, K. Vasudeva Shettigar ${ }^{3}$ \\ PG Scholar, NMAMIT, Nitte, Karkala, India ${ }^{1}$ \\ GOEPEL electronics India Pvt Ltd, India ${ }^{2}$ \\ Assistant Professor, NMAMIT, Nitte, Karkala, India ${ }^{3}$
}

\begin{abstract}
Instrument cluster is the Human Machine Interface (HMI) in vehicle which ensures the safety and comfort of the driver by showing the prevailing condition of the vehicle. These days instrument clusters are getting more and more complex. So the testing of instrument cluster for proper functioning i.e. HMI, indicators and warning signals has to be conducted. This paper presents the cost effective and efficient method of testing the instrument cluster using the software developed in LabVIEW and CAN protocol. CAN controller interfaces the PC and instrument cluster, it sends signals through CAN bus to test the tell-tale, dials, fuel gauges and display's in the cluster.
\end{abstract}

Keywords: LabVIEW, CAN communication, Instrument cluster, TOMLine.

\section{INTRODUCTION}

In the recent years the automotive segments has become sophisticated, the instrument cluster which assist the driver with information of driving condition has become more complex, so in the process of manufacturing it should be tested to ensure its proper functioning. In some automobile company unit testing is performed manually, using traditional instruments. This is tedious as the number of functions in the cluster increases.

These days people search for ways to reduce their time and effort to do any job. Automatic testing is more efficient and reliable, and less prone to human errors due to negligence and fatigue. Hardware parts increase the risk of errors during the production, thus the testing of the system has to be carefully performed. For that reason, automated testing in production lines is important to reduce the risk of hardware fault. This has led to the implementation of this project.

The software for automated instrument cluster testing is developed in LabVIEW. LabVIEW platform has the capability of data acquiring, monitoring, processing and controlling. CAN is one of the widely used communication interface in the automobile. It reduces the complexity of wiring and improves data transfer reliability. CAN controller is a vital device in the system to process the message received from PC. It provides dynamic signals to the system under test.

An automated test based on LabVIEW application, for units testing of MIKME advanced microphone in a serial production is proposed, which is 20 times faster than manual test [1]. Currently, automated design validation testing of a vehicle instrument cluster using dSPACE midsize simulator is presented [2]. An automated test system for various systems using LabVIEW is implemented over last few years $[3,4]$. Automotive instrument network design and data transmission using CAN bus [5], characteristics and message transmission rules of CAN bus using different processors and controllers [6] are proposed in recent years.

The proposed work in this paper explains the validation test of vehicle instrument clusters in real-time using LabVIEW application, on successfully performing the test, the series production of the cluster is started.

\section{APPLICATION DESIGN}

The designed LabVIEW application for cluster testing mainly has four panels. First panel we see when we open the application is shown in Fig. 1. It is the main panel or front panel, where we can select the required subpanel by pressing the respective buttons on the main panel. The buttons in main panel are Test, Parameter, Settings, About, Exit. The table in the main panel shows the information about the tasks performed in the application with date and time and exit button will close the application. The necessary parameters for interface are given in settings panel Fig. 2. It includes selecting the type of test to be performed either End of Line Test (EOL) or Endurance Test, Number of test cycles to be performed and number of cycles to be skipped which are applicable for endurance test, and CAN port, TOMLine file paths also should be entered. TOMLine is the Vision software of Goepel electronics for analysing the cluster image.

In the parameter panel shown in Fig. 3. we give the name of the test in test step table (eg: Ignition, Right indicator) and by pressing add button we add the test case. The conditions for the test cases should be given in subsequence table (eg: on or off). A test case can have any number of subsequence. For every subsequence the 
general setting, CAN setting and TOMLine setting has to analysed by TOMLine and the test result is displayed in be entered and saved. The selected test case or the table of test panel. Current cycle, number of cycle and subsequence can be deleted by pressing delete button, on skip cycle are indicators, which show the values entered in pressing execute button test page will open, and the back the settings panel if endurance test is performed, and it button will take us to the front panel.

will be zero for EOL test. Refresh and cancel button will

When the test button in main panel is pressed it will take start the test from beginning and stop the test respectively. us to test panel which is shown in Fig. 4. Here we perform On pressing the report button, html report as shown in Fig. the test for the test cases entered in the parameter panel by 5 . is generated for the test results displayed in the table of pressing start button. The test is conducted in the order Test panel and the generated report is saved for the future given in parameter page. Cluster image for every case is reference.

displayed in the picture box, and the same image is

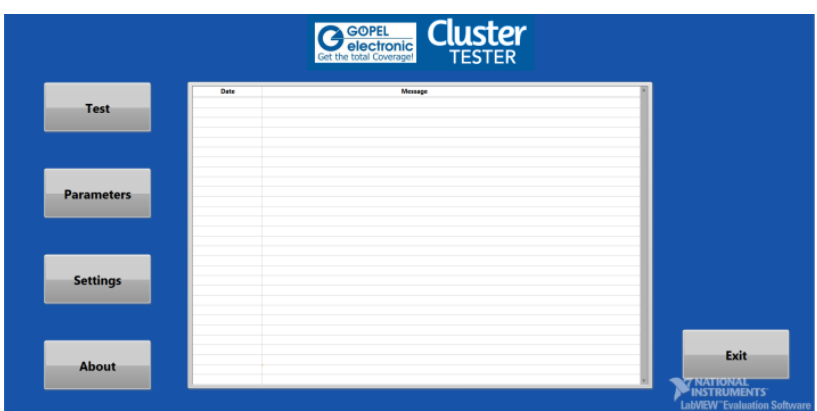

Fig. 1. Main Panel

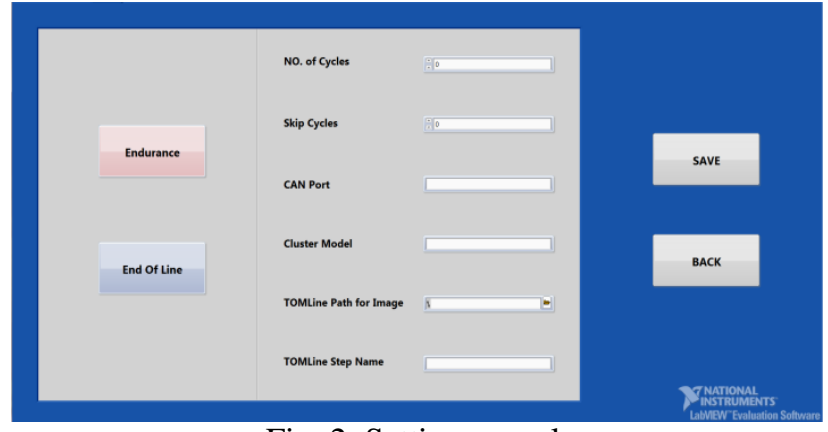

Fig. 2. Settings panel

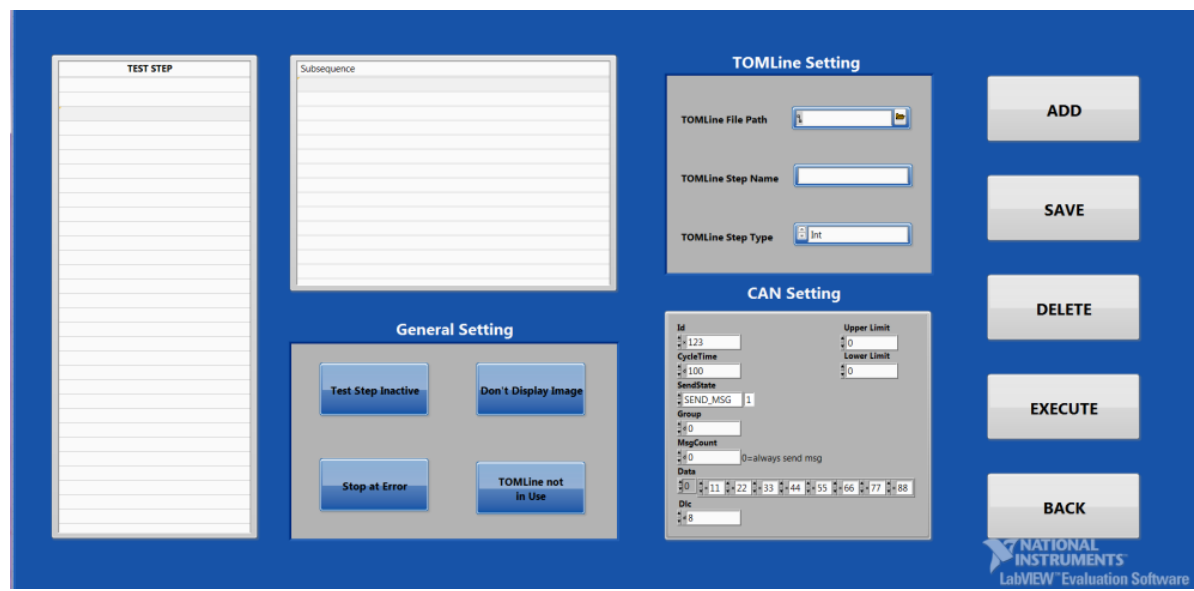

Fig. 3. Parameter panel

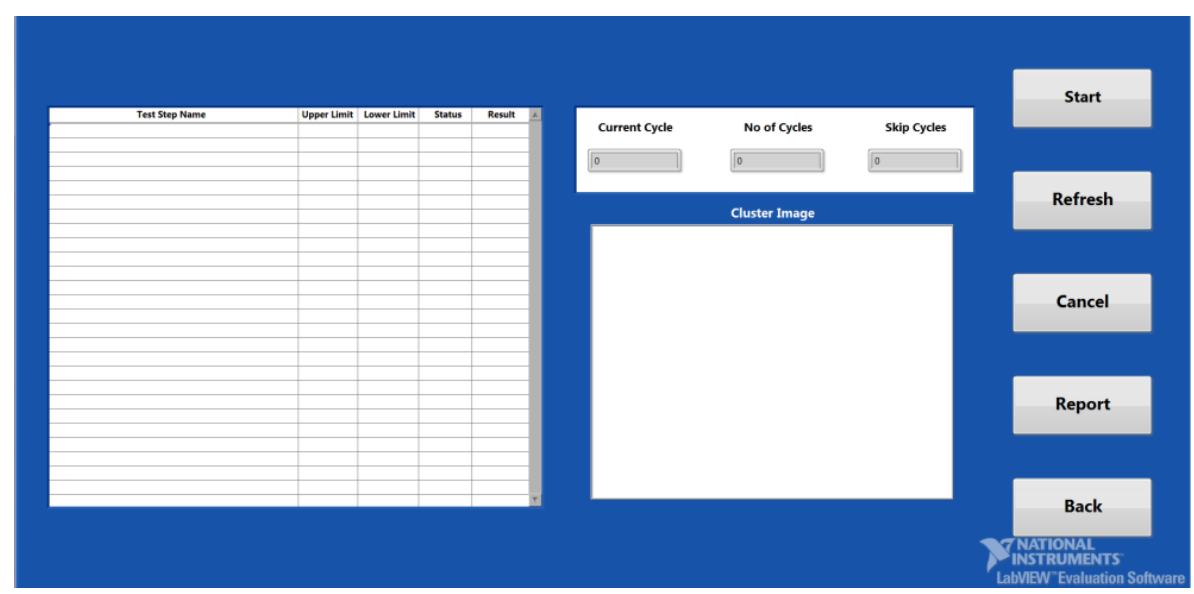

Fig. 4. Test panel 


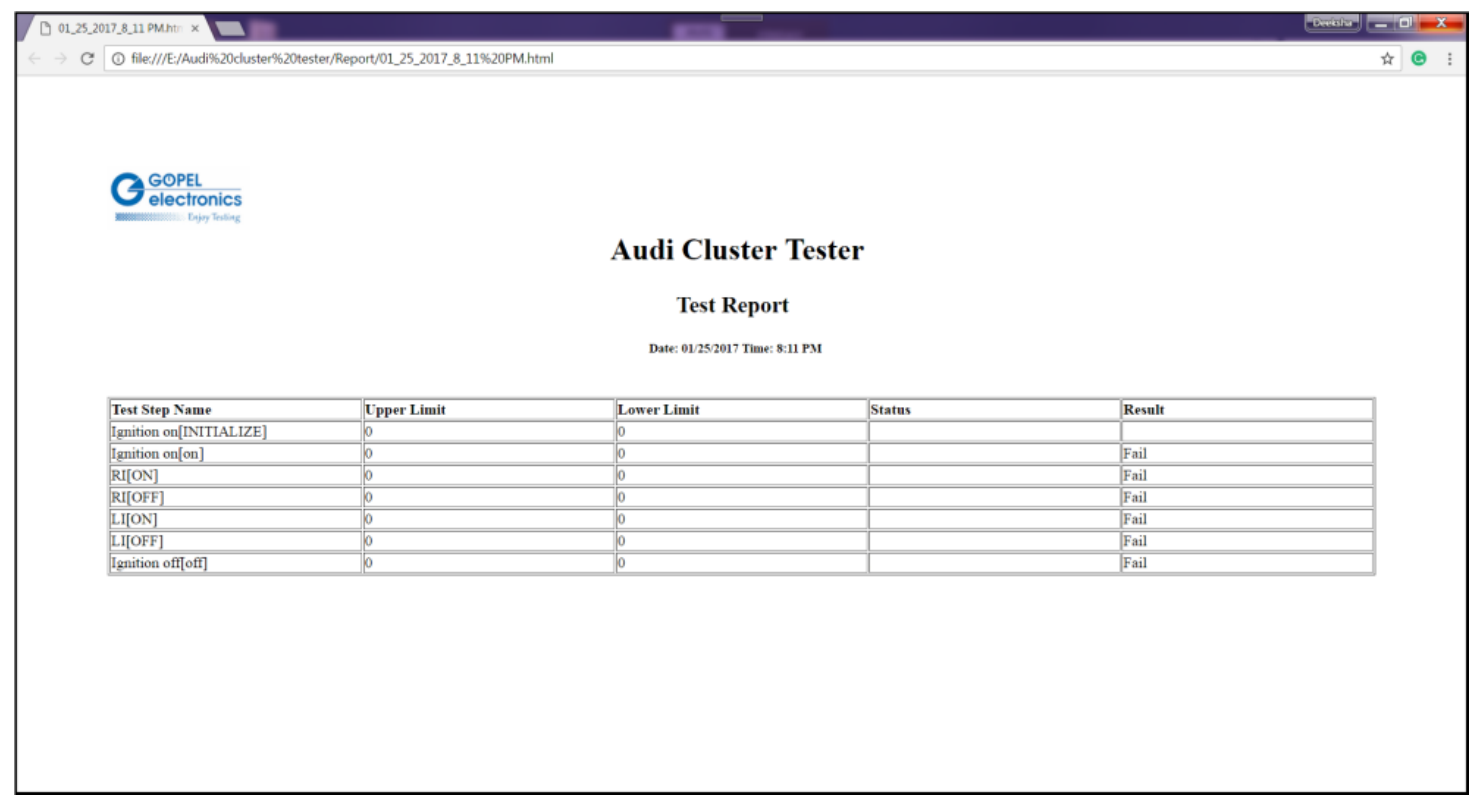

Fig. 5. Generated html report

\section{III.TEST SETUP AND WORKING PRINCIPLE}

The test system for testing the instrument cluster is shown in Fig.6, it consists of host PC with LabVIEW application, unit under test and communication interfaces.

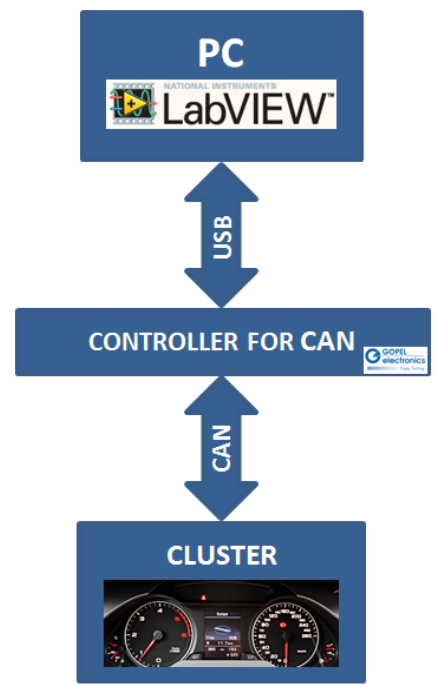

Fig. 6. Automated control of Instrument cluster using LabVIEW application

\section{A. LabVIEW}

The main controller of the system is designed LabVIEW application. LabVIEW is a graphical programming language. So we can develop better programs in LabVIEW since graphical programs are easier to maintain, edit and understand. We can reuse the code in different operations hence it is simpler to program and easy to understand just by looking at the code.
B. Device Under Test

The device under test is the instrument cluster shown in Fig. 7. it is one of the most complex electronic embedded systems in vehicles. Its functionality is distributed among many ECUs. The cluster consists of tell-tale, warning indicators, fuel gauge, speedometer, tachometer, and LCD display.

The major components of instrument cluster which are tested include Ignition, Right and Left Indicators, Parking Light, Door Open Warning, Bonnet and Boot Lid Open Warning, Low Fuel Level Warning, Tyre Pressure Monitoring, Power Steering System, Speedometer gauge, Fuel gauge.

\section{CAN}

CAN protocol is used for communication between PC and instrument cluster. CAN frame which carry CAN messages consist of an ID, a varying number of data bytes, and data length specified by DLC. The particular function in instrument cluster will have unique ID number and it can have any number of data bytes. The size of different fields in CAN frame is given below.

- ID: Identifier: 11 or 29 bits

- Data Bytes: 0 - 8 bytes

- $\quad$ DLC: Data Length Code: 4 bits -8 bits

CAN information given by user is automatically handled by the CAN controller i.e. forming CAN frames for the given CAN details and gets the message on the bus.

LabVIEW application acts as front end interface in testing the device under test. The designed application is generic since the user has got the freedom to add, modify or delete the test cases. 


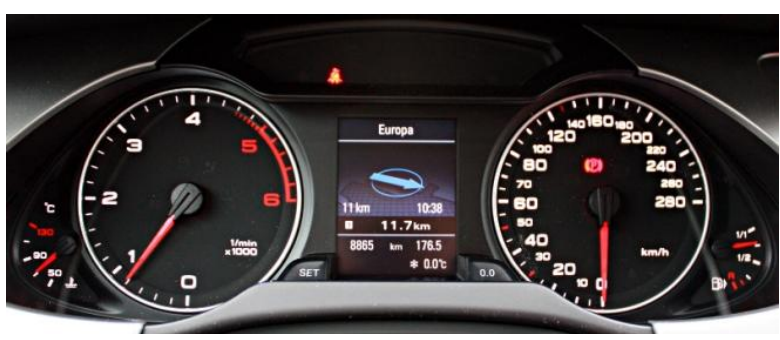

Source: Internet

Fig. 7. Instrument cluster

The CAN message of the respective test cases are sent to the DUT using CAN API (Application Program Interface) in LabVIEW. The basic CAN API function block consists of an initialization, start, read or write data and clear.

When the test is performed respective CAN message of the test cases are passed through USB cable to CAN controller, where the message is converted to CAN frames and sent to Instrument cluster through CAN bus. The instrument cluster on receiving the signal performs the respective functions. The result of the test observed in cluster is as shown in Fig. 8. Once the test is successfully performed; the series production of the same is started.

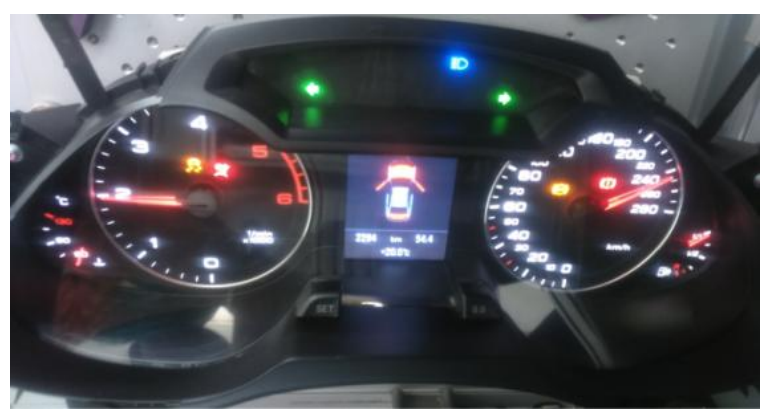

Fig. 8. Instrument cluster showing various indicators

\section{IV.CONCLUSION AND FUTURE WORK}

The instrument cluster test system acts as virtual vehicle to test the clusters behavior and to test the functionality.

LabVIEW application is successfully applied for testing the instrument cluster. Using this system, we can saves time, reduces complexity and error in testing. This will save time and money.

The machine vision system to read and analyze the test result will be done as future work.

\section{ACKNOWLEDGEMENT}

I would like to show my gratitude to ATS team of GOEPEL electronics India Pvt Ltd for their selfless help and NMAM Institute of Technology for all support and encouragement in doing this project.

\section{REFERENCES}

[1] Vladimir D. Ćatić, Natalija M. Lukić, Iva M. Salom, Vukašin P. Ristić, Milenko M. Kabović, and Nikola M. Nenadić, "An Automated Environment for Hardware Testing Using PXI Instrumentation and LabVIEW Software," 24th Telecommunications forum TELFOR 2016 Serbia, Belgrade, November 22-23, Nov 2016 IEEE

[2] Yingping Huang, Alexandros Mouzakitis, Ross McMurran, Gunwant Dhadyalla and R. Peter Jones, "Design Validation Testing of Vehicle Instrument Cluster Using Machine Vision and Hardware-in-the-loop," in Proc. IEEE International Conference on Vehicular Electronics and Safety,Columbus, OH, USA September 22-24, 2008.

[3] Amit Dhondiram Magdum, A. A. Agashe, "Monitoring and controlling the industrial motor parameters remotely using LabVIEW," in IEEE International Conference On Recent Trends In Electronics Information Communication Technology, May 20-21, 2016, India.

[4] Jiayue Li, Zhaoqin Peng, and Maidan Luo, "Testing Methods for Performance of Steering Gear System Based on LabVIEW," in Proceedings of 2016 IEEE Chinese Guidance, Navigation and Control Conference August 12-14, 2016 Nanjing, China.

[5] Jiejie Dai and Hui Song, "Design And Realization Of Can Bus Vehicle Instrument Cluster Based On M C/Os-Ii,"

[6] Bin Ling, Fengchao Peng andAilan Li, "The Car Body Control Bus Design Based On Can/Lin Bus," IEEE 2011.

[7] National Instruments Corporation 1-1 Controller Area Network (CAN) Tutorial

\section{BIOGRAPHIES}

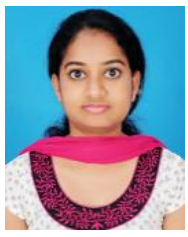

Deeksha K, received her B.E. (E\&E) degree from KVG College of Engineering India in 2015. She is pursuing her M. Tech (Microelectronics and Control Systems) in Nitte Mahalinga Adyanthaya Memorial Institute of Technology, Nitte, Karkala, India, under Visvesvaraya Technological University, Belgaum. Her area of interests includes Control systems, Automation and Electronics.

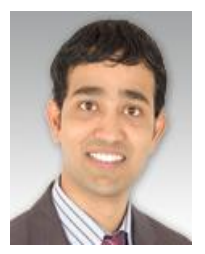

Sandeep Reddy Cherukupalli, received his B.E. degree in Electrical and Electronics from KVG College of Engineering, Sullia under Visvesvaraya Technological University, Belgaum, India in 2005 and MS degree in Microelectronics from Darmstadt University of Applied Science, Darmstadt, Germany in 2008. He is currently working for ATS department in GOEPEL electronic India Pvt. Ltd. His area of interest includes Automation in Automotive, Microcontrollers, Embedded C, Embedded Systems. He was an intern at Fraunhofer-Institute where he worked for Testing and Validation of Web Service for enterprise application integration (EAI). Analysis of Enterprise Service Bus (ESB). An European Union project in 2007 and at Gleichmann \& Co Electronics, Gmbh in 2008, where he developed a high speed data transfer protocol between two 
Vol. 5, Special Issue 2, April 2017

stratix II FPGA's. He worked as Sales Manager at Smart electronics development $\mathrm{Gmbh}$ for 2 years 10 months.

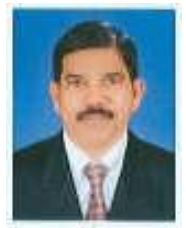

K. Vasudeva Shettigar, received his B.E. (E\& E) degree from SJCE, Mysore University, Mysore, India in 1986, and M.Tech. (Power Systems) degree in 1992 from National Institute of Engineering affiliated to Mysore University. Presently Mr. Vasudeva Shettigar is serving as Associate Professor, Dept. of Electrical \& Electronics Engineering, Nitte Mahalinga Adyanthaya Memorial Institute of Technology, Nitte, Karkala, India. He is Life member of ISTE. He has published 11 technical research papers in various National and International conferences. He has chaired International conference held at NMAMIT Nitte during May 2011, 2013 and 2015. Mr. Shettigar has organized several National Conferences and National Workshops and Short term training programes (STTP) in the college for the benefit of faculty (NMAMIT) and faculty of neighboring Institutes. His areas of interest include power system \& protection, power quality and High Voltage Engineering. He has a teaching experience of over 27 Years, and guided over 50 U.G \& 24 P.G. Technical Project works. 